\title{
Open/closed string duality is incompatible with renormalization in 't Hooft large- $N$ QCD, but not in Veneziano large- $N$ QCD
}

\author{
Marco Bochicchio* \\ INFN sez. Romal, \\ Piazzale A. Moro 2, Roma, I-00185, Italy \\ E-mail: marco.bochicchio@romal.infn.it
}

\begin{abstract}
Solving 't Hooft or Veneziano large- $N$ QCD by a string theory is a long-standing problem. The would-be canonical string solution for 't Hooft large- $N$ QCD is a theory of closed strings for the glueballs and of open strings for the mesons. Recently, we demonstrated that an inconsistency arises between the renormalization properties of the 't Hooft large- $N$ QCD S matrix, worked out in Phys. Rev. D 95, 054010, and the open/closed duality of the would-be string solution, which thus does not exist in the canonical string framework. Specifically, the glueball amplitudes in the would-be open-string one-loop effective action, which canonically only get contributions from surfaces with one hole, must be ultraviolet (UV) divergent by the aforementioned renormalization properties. Hence, naively, the inconsistency occurs because these amplitudes are dual to tree closed-string diagrams, which are universally believed to be both UV finite - since they are closed-string tree diagrams - and infrared finite because of the glueball mass gap. In fact, the inconsistency follows from a low-energy theorem of the Novikov-Shifman-Vainshtein-Zakharov type, derived in Phys. Rev. D 95, 054010, that controls the renormalization in QCD-like theories. Interestingly, the proof of the inconsistency, at least in its present form, does not extend to a would-be string solution for Veneziano large- $N$ QCD. In an a-posteriori-related development, we proposed a new class string theories on noncommutative twistor space, whose space-time one-loop effective action in the open-string sector gets contributions, contrary to the canonical framework, from string instantons wrapping geometrically planar surfaces with any number of holes, topologically as in the Veneziano-planar expansion, avoiding the inconsistency.
\end{abstract}

The European Physical Society Conference on High Energy Physics

5-12 July, 2017

Venice

${ }^{*}$ Speaker. 


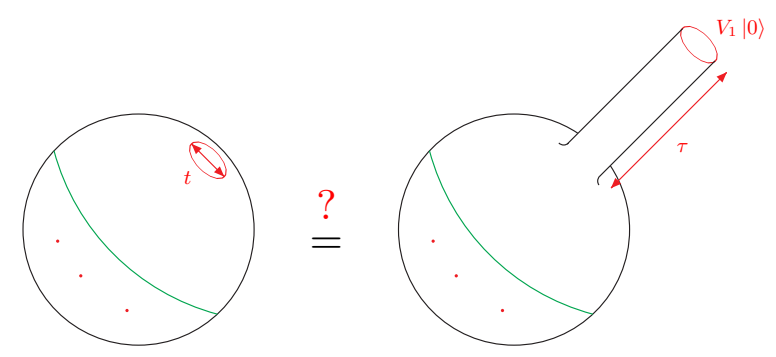

Figure 1: If a canonical string solution for 't Hooft large- $N$ QCD exists, the one-loop (hole) open-string diagram in the lhs is a cutoff-independent volume form on the moduli that, by integrating on the moduli, e.g., $t$, must be UV log divergent because of the renormalization properties of large- $N$ QCD. By open/closed duality the lhs coincides, as a volume form on the moduli, with the tree closed-string diagram in the rhs, where the glueballs propagate in the cylinder of length $\tau=f(t), \tau \rightarrow \infty$ as $t \rightarrow 0$. Thus, the rhs must be divergent, but only by integrating on the moduli, e.g., $\tau$, that is incompatible with the universal belief that is both UV finite - since it is a tree closed-string diagram - and IR finite because of the glueball mass gap. In fact, a low-energy theorem (Sec. 2) in large- $N$ QCD implies that the rhs is log divergent before integrating on $\tau$ because of the explicit UV log divergence of the operator counterterm, $V_{1}$, in the boundary state, $V_{1}|0\rangle$. Hence, the rhs cannot coincide as a volume form on the moduli with the lhs, and the open/closed duality cannot hold in 't Hooft large- $N$ QCD.

\section{Introduction and conclusions}

Solving the 't Hooft [1] or the Veneziano [2] large- $N$ expansion of QCD, with gauge group $S U(N)$ and $N_{f}$ quark flavors, is a long-standing problem with outstanding implications for the theory of strong interactions. In both expansions there is a correspondence [1,2] between Riemann surfaces and sums of Feynman graphs triangulating the very same topological surfaces arising from the perturbative expansion in terms of 't Hooft coupling, $g^{2}=g_{Y M}^{2} N$. In 't Hooft large- $N$ QCD, the leading contribution at large $N$ is the 't Hooft-planar (tHp) theory [1], which consists of planar diagrams with the topology of a punctured sphere in the glueball sector, or of a disk with punctures on the boundary in the meson sector. In Veneziano large- $N$ QCD, the leading contribution is the Veneziano-planar $(\mathrm{Vp})$ theory [2], which consists of the geometrically planar diagrams, e.g., of an infinite sum of hole insertions in a punctured sphere, or in the aforementioned punctured disk. The hole boundaries arise perturbatively from the quark loops in the quark functional determinant.

This topological matching has been suggesting the existence of a canonical string solution [2, 3] for 't Hooft large- $N$ QCD, of closed-strings for the glueballs and of open strings for the mesons $[1,2]$, and, more generally, for confining asymptotically free QCD-like theories. Yet, it has not been found despite the advent of the celebrated gauge/gravity duality [4] in the string framework. The key ingredient of the canonical string ansatz, in addition to the topological matching with the Feynman diagrams, is the existence of an auxiliary conformal field theory living on the string world-sheet of fixed topology [3], which is employed to define the $S$-matrix amplitudes [3] in the would-be string solution for 't Hooft or, eventually, for Veneziano large- $N Q C D$. The existence of the conformal structure has far-reaching consequences [3], and it is not at all obvious that is in fact compatible with the structure of large- $N$ QCD, despite its compatibility has been taken for granted [4] for the past forty years.

Recently, we investigated the renormalization properties [5] of the large- $N$ Yang-Mills (YM) 
theory and QCD: the renormalization group (RG) and asymptotic freedom (AF) imply that the large- $N$ YM S matrix is ultraviolet (UV) finite, while nonplanar contributions to the $S$-matrix, in both the large- $N$ QCD 't Hooft and Veneziano expansions, are UV divergent though renormalizable.

The UV finiteness of the large- $N$ YM S matrix [5] matches the universal belief that all the consistent closed-string theories are UV finite [6], and thus it is compatible with the existence of its would-be canonical string solution [5]. Yet, we demonstrated that the open/closed string duality [7], a direct consequence of the string conformal symmetry, is incompatible with the nonplanar renormalization in 't Hooft large- $N$ QCD $[8,9]$.

It follows a no-go theorem [8,9] recalled in Fig. 1 and Sec. 2: RG and AF are incompatible with the open/closed duality in a would-be canonical string solution - which thus does not exist in the canonical framework - for 't Hooft large- $N$ QCD.

Firstly, in this paper we make a similar investigation on the Veneziano expansion. Interestingly, we find that the proof of the inconsistency of the open/closed duality with the large- $N$ renormalization, at least in its present form, does not apply to the Veneziano expansion of QCD and QCD-like theories (Sec. 3). Secondly, we briefly outline a noncanonical way-out $[8,9]$ of the no-go theorem, by constructing [10] a new class of open/closed string theories on noncommutative twistor space: the space-time one-loop S matrix in the open-string sector receives noncanonical contributions from string instantons wrapping geometrically planar surfaces with any number of holes $[8,9]$, topologically as in the Vp expansion, avoiding the inconsistency [8, 9] (Sec. 4).

\section{The no-go theorem on the canonical string in 't Hooft large-N QCD}

Since the tHp S matrix only involves tree-level interactions [11, 12], it must be UV finite. In massless QCD the UV finiteness is obtained by the planar renormalization of $g$, in such a way that the planar S matrix is UV finite once it is expressed [5] in terms of the planar RG invariant, $\Lambda_{Q C D}^{P}$. In massive $\mathrm{QCD}$, the renormalized quark masses, $m^{P}$, also occur as parameters.

The planar S matrix is not unitary though, since it consists of tree amplitudes. To restore unitarity [1, 2], non-tHp diagrams, which carry handles and holes and are subleading in $\frac{1}{N}$, must be included. In the perturbative QCD S matrix, non-tHp quark loops from the quark functional determinant are UV divergent, and modify the scheme-independent first coefficient of the planar beta function. Thus, these divergences cannot be reabsorbed nonperturbatively in a finite renormalization of $\Lambda_{Q C D}^{P}$ [5].

Specifically, the non-tHp diagram in the lhs of Fig. 1, which renormalizes at order of $\frac{N_{f}}{N}$ the $k$-glueball tree amplitude, is UV log divergent: perturbatively because of the quark loop (i.e., the hole boundary), and nonperturbatively because of the coupling with the mesons [5].

For the diagrams in Fig. 1 the open/closed duality of the would-be string solution implies:

$$
\left.\left\langle D_{k}\left(m_{i}\right)\right| \text { Annulus }(t)\right\rangle d m_{1} \wedge \cdots \wedge d m_{i} \wedge \frac{d t}{t}=\left\langle D_{k}\left(m_{i}\right)\left|\exp \left(-\tau H_{\text {Closed }}\right) V_{1}\right| 0\right\rangle d m_{1} \wedge \cdots \wedge d m_{i} \wedge d \tau
$$

for a map, $\tau=f(t)$, such that $\tau \rightarrow \infty$ as $t \rightarrow 0$ [7, 13]. $m_{1} \cdots m_{i}$ are the remaining moduli of the surface obtained gluing $D_{k}$, a disk with $k$ punctures in the interior, to an annulus with modulus $t$ (i.e., a disk with one hole) in the open-string side of the duality (lhs), or to a cylinder with modulus $\tau$ in the closed-string side (rhs). $H_{\text {Closed }}$ is the world-sheet Hamiltonian in the closed sector. 
If the QCD canonical string solution exists, after integrating on the moduli, Eq. 2.1 implies the equality between (amputated on-shell) glueball $k$-point correlators in large- $N \mathrm{QCD}$, for a certain zero-momentum, possibly non-local, scalar gauge-invariant pure-glue operator, $V_{1}$ :

$$
\left\langle\operatorname{Tr} F^{2} \cdots T r F^{2}\right\rangle_{\text {conn }}^{1-\text { OpenStringLoop }}=\left\langle\operatorname{TrF}^{2} \cdots T r F^{2} V_{1}\right\rangle_{\text {conn }}^{\text {TreelosedString }}
$$

within the leading $\frac{1}{N}$ accuracy. In the canonical string framework both sides of Eq. 2.2 may only be divergent after integrating on the moduli in Eq. 2.1, specifically on $t$ and $\tau$, since the integrands are UV cutoff $(\Lambda)$ independent and pointwise finite on their definition domain, though not necessarily integrable functions.

In the would-be large- $N$ QCD string solution there is a tension between the UV log divergence of the $k$-glueball amplitude coupled to mesons in the lhs of Eq. 2.2 and the universally believed UV finiteness [6] of the geometrically planar [7] diagram in the rhs of Eq. 2.2 which, being both a tree and a closed-string diagram, should be UV finite.

The canonical formulation of string theory resolves this tension in the general framework [7] and in solvable examples that bear deep analogies with the issue considered here [13]: the UV divergence of the open-string diagram in the lhs is mapped by open/closed duality into an infrared (IR) divergence of the dual tree closed-string diagram [7, 13] in the rhs of Eq. 2.2, according to the relation $\tau \rightarrow \infty$ as $t \rightarrow 0$ in Eq. 2.1. Yet, the IR divergence may only occur if the tree closed-string theory has no mass gap. Therefore, this cannot work for large- $N$ QCD $[8,9]$ if it is assumed that large- $N$ QCD has a mass gap in the tHp glueball sector.

Therefore, assuming that the closed-string solution provides an UV finite tree diagram in the rhs of Eq. 2.2 as it is universally believed [6], we get immediately the first no-go theorem [8]: the open/closed duality in a would-be canonical string solution for the 't Hooft large- $N$ QCD S matrix is incompatible with RG, AF and the mass gap in the tHp glueball sector.

However, by assuming the mass gap, the only logically possible alternative is that the rhs of Eq. 2.2 may be UV log divergent rather than IR log divergent. Accordingly, the potential inconsistency would be resolved by contradicting the universally believed UV finiteness of the tree closed-string diagrams. We find out what actually happens in 't Hooft large- $N$ QCD computing explicitly $V_{1}$ by means of a low-energy theorem of the Novikov-Shifman-Vainshtein-Zakharov type in [5]:

$$
\begin{aligned}
\left\langle\operatorname{Tr} F^{2} \cdots \operatorname{Tr} F^{2}\right\rangle_{d i v}^{N P} & =\frac{N \beta^{P}(g) \Lambda_{Q C D}^{N P}}{g^{3} \Lambda_{Q C D}^{P}} \int\left\langle\operatorname{Tr} F^{2} \cdots \operatorname{Tr} F^{2}\right\rangle^{P}\left\langle\operatorname{Tr} F^{2}(x)\right\rangle^{P}-\left\langle\operatorname{Tr} F^{2} \cdots \operatorname{Tr} F^{2} \operatorname{Tr} F^{2}(x)\right\rangle^{P} d^{4} x \\
& \equiv\left\langle\operatorname{Tr} F^{2} \cdots \operatorname{Tr} F^{2}\left[V_{1}^{P}\right]_{d i v}\right\rangle^{P}-\left\langle\operatorname{Tr} F^{2} \cdots \operatorname{Tr} F^{2}\right\rangle^{P}\left\langle\left[V_{1}^{P}\right]_{d i v}\right\rangle^{P}
\end{aligned}
$$

Eq. 2.3 has precisely the structure to match Eq. 2.2, and it computes its divergent parts in both sides [5] due to the renormalization of $\Lambda_{Q C D}^{P}$, with $\left[V_{1}^{P}\right]_{d i v}=-\frac{N \Lambda_{Q C D}^{N P}}{\Lambda_{Q C D}^{P}} \frac{\beta^{P}(g)}{g^{3}} \int T r F^{2} d^{4} x=N\left[\beta_{0}^{N P} \log \left(\frac{\Lambda}{\Lambda_{C C D}^{P}}\right)+\right.$ $\cdots] \int \operatorname{Tr}^{2} d^{4} x$. Moreover, $V_{1}$ can be derived in large- $N$ QCD, $V_{1}=N_{f} \operatorname{Tr} \log \frac{i \gamma_{\mu} D^{\mu}(A)-Z_{m}^{P} m^{P}}{i \gamma_{\mu} D^{\mu}(0)-Z_{m}^{P} m^{P}}=$ $N \beta_{0}^{N P} \log \left(\frac{\Lambda}{\Lambda_{Q C D}^{P}}\right) \int \operatorname{Tr}^{2} d^{4} x+$ nonlocal UV finite terms, directly from its definition in Eq. 2.2, by expanding the QCD action at the order of $\frac{N_{f}}{N}$, with $Z_{m}^{P}$ defined by the expansion, $Z_{m} \sim\left[\log \left(\frac{\Lambda}{\Lambda_{Q C D}}\right)\right]^{-\frac{\gamma_{0} m}{2 \beta_{0}}}$ $\sim Z_{m}^{P}(1+\cdots), \beta_{0}=\beta_{0}^{P}+\beta_{0}^{N P}$ and $\gamma_{0 m}=\frac{3}{(4 \pi)^{2}}\left(1-\frac{1}{N^{2}}\right)$, where the dots both in $Z_{m}$ and in $\left[V_{1}^{P}\right]_{d i v}$ stand for subleading UV log-log divergences [5]. Remarkably, within the leading-log accuracy, $-\left[V_{1}^{P}\right]_{d i v}=-\left[V_{1}\right]_{d i v}$ is the local counterterm, due to coupling with mesons [5] or to quark loops 
from the quark functional determinant, that produces the non-tHp correction to $\beta_{0}^{P}$ [5]. Besides, the insertion of $\int T r F^{2} d^{4} x$ in the rhs of Eq. 2.2 is UV finite by the operator product expansion and AF [9]. Thus, the UV finite non-local terms in $V_{1}$ may contribute in the rhs of Eq. 2.2 at most UV log-log divergences.

The obstruction to the open/closed duality is the UV log divergence of the local part in $V_{1}$ before its insertion in the rhs of Eq. 2.2, as opposed to the log-log divergences that occur after the insertion. Indeed, by transferring into the would-be string solution the QCD result for $V_{1}$ in the rhs of Eq. 2.1, $\left\langle D_{k}\left(m_{i}\right)\left|\exp \left(-\tau H_{\text {Closed }}\right) V_{1}\right| 0\right\rangle$ is $\Lambda$ dependent and, as a function of the moduli, pointwise UV log divergent as $\Lambda \rightarrow \infty$, since $V_{1}|0\rangle$ is created from the closed-string vacuum, $|0\rangle$, by an operator, $V_{1}$, that contains a log-divergent counterterm.

This contradicts the $\Lambda$ independence and pointwise finiteness of the integrands in both sides of Eq. 2.1. A similar argument holds for $\mathscr{N}=1$ SUSY QCD [5]. A second stronger no-go theorem [9], based only on UV arguments, follows: RG and AF are incompatible with the open/closed duality in a would-be canonical string solution - that thus does not exist - for the 't Hooft large$N$ QCD S matrix. The incompatibility extends to a vast class [5] of 't Hooft large- $N$ QCD-like theories including $\mathscr{N}=1$ SUSY QCD.

\section{The no-go theorem does not apply to the string in Veneziano large-N QCD}

The proof of the second no-go theorem does not apply to the would-be string in the Veneziano expansion of QCD and QCD-like theories. The non-Vp diagrams may only be UV log-log divergent [5], since the divergences arise from the non- $\mathrm{Vp}$ contribution to the second coefficient of the beta function [5]. Hence, the $\log$ divergence and $\Lambda$ dependence of the operator $V_{1}$ is reabsorbed by the $\mathrm{Vp}$ renormalization [5] invoving the infinite sum over the holes. It only remains the log$\log$ divergence due to the overlapping of the Vp-renormalized $V_{1}, V_{1}^{V P}$ ren, with a handle [5] in a suitable version of Eq. 2.2. But this log-log divergence only occurs after taking the v.e.v. of the $\Lambda$-independent nonlocal operator, $V_{1}^{V P}$ ren , in the rhs of the suitable version of Eq. 2.2.

Thus, the $\Lambda$-independence of $V_{1}^{V P}$ ren is compatible with the pointwise finiteness of the string integrands in both sides of a suitable version of Eq. 2.1. Thus, no contradiction with the open/closed duality necessarily arises.

\section{Noncommutative twistor strings as noncanonical strings}

In an a-posteriori-related development, we proposed certain noncommutative twistor strings $[10,14]$ as noncanonical candidates for the 't Hooft large- $N$ QCD S matrix. The open-string oneloop effective action arises as a functional determinant which is the limit of the sum on a lattice of world-sheet instantons, according to the identity [8]:

$$
\Gamma=\sum_{p} \operatorname{Tr} \log \left(1-P \exp i \int_{C_{p}} \hat{B}_{\lambda}\right)=\left.\sum_{p} \hat{\operatorname{T}} r \log \left(\frac{d}{d \lambda}+\hat{B}_{\lambda}\right)\right|_{C_{p}}=\log \operatorname{Det}\left(\frac{d}{d \lambda}+\hat{B}_{\lambda}\right)
$$

Indeed, each term in the expansion of the first log in Eq. 4.1 is due to the insertion of Wilson loops, i.e., of string instantons wrapping Lagrangian submanifolds with any number of boundaries, topologically as in the Vp expansion. The space-time one-loop $S$-matrix [10] arises evaluating $\Gamma$ 
around the nontrivial saddle point, $\tilde{B}_{\lambda}: \tilde{B}_{p^{+}}=-\hat{P}_{-}+\frac{\left(\frac{1}{2} \Lambda_{Q C D}^{2}\right)^{-1} \hat{P}_{\mu_{u}} \hat{P}_{\bar{u}}-\hat{P}_{-} \frac{d}{d \hat{P}_{-}}+Q}{\frac{P^{+}}{2} \Lambda_{Q C D}^{2}}$, where $\lambda=\frac{\hat{P}_{+}}{\Lambda_{Q C D}^{2}}$ is identified with the light-cone momentum operator. It follows [10]:

$$
\Gamma=\log \operatorname{Det}\left(\hat{P}_{+} \hat{P}_{-}-\hat{P}_{u} \hat{P}_{\bar{u}}-\frac{1}{2} \Lambda_{Q C D}^{2}\left(\hat{P}_{+} \frac{d}{d \hat{P}_{+}}-\hat{P}_{-} \frac{d}{d \hat{P}_{-}}+Q\right)+\delta B_{p+}\right)
$$

with the spectrum [14] of the square mass operator determined by the spin operator, $\hat{P}_{+} \frac{d}{d \hat{P}_{+}}-\hat{P}_{-} \frac{d}{d \hat{P}_{-}}$, and by $Q$, which turns out to be integral or semi-integral valued for cohomological reasons, arising from the infinite noncommutative Hodge structure of the theory [10]. Because of the noncanonical definition of the amplitudes, no potential inconsistency with the large- $N$ renormalization occurs.

\section{Acknowledgments}

We would like to thank Gabriele Veneziano for the helpful comments.

\section{References}

[1] G. 't Hooft, A planar diagram theory for strong interactions, Nucl. Phys. B 72 (1974) 461.

[2] G. Veneziano, Some Aspects of a Unified Approach to Gauge, Dual and Gribov Theories, Nucl. Phys. B 117 (1976) 519.

[3] G. Veneziano, An Introduction to Dual Models of Strong Interactions and their Physical Motivations, Phys. Rep. 9 (1974) 199.

[4] O. Aharony, S. S. Gubser, J. Maldacena, H. Ooguri, Y. Oz, Large-N Field Theories, String theory and Gravity, Phys. Rept. 323 (2000) 183, arXiv:hep-th/9905111.

[5] M. Bochicchio, The large-N Yang-Mills S matrix is ultraviolet finite, but the large-N QCD S matrix is only renormalizable, Phys. Rev. D 95 (2017) 054010, arXiv:1701.07833 [hep-th].

[6] E. Witten, What every physicist should know about string theory, Phys. Today 68 (2015) no. 11, 38.

[7] J. Khoury, H. Verlinde, On Open/Closed String Duality, Adv. Theor. Math. Phys. 3 (1999) 1893, arXiv:hep-th/0001056.

[8] M. Bochicchio, Asymptotic Freedom versus Open/Closed Duality in large-N QCD, arXiv:1606.04546 [hep-th].

[9] M. Bochicchio, Renormalization in large-N QCD is incompatible with open/closed string duality, arXiv:1703.10176 [hep-th].

[10] M. Bochicchio, An Asymptotic Solution of Large-N QCD, for the Glueball and Meson Spectrum and the Collinear S-Matrix, In: Proceedings, 16th International Conference on Hadron Spectroscopy (Hadron 2015), AIP Conf. Proc. 1735 (2016) 030004, DOI: 10.1063/1.4949387.

[11] A. Migdal, Multicolor QCD as a dual-resonance theory, Annals of Physics 109 (1977) 365.

[12] E. Witten, Baryons in the 1/N Expansion, Nucl. Phys. B 160 (1979) 57.

[13] P. Di Vecchia, A. Liccardo, R. Marotta, F. Pezzella, On the Gauge/Gravity Correspondence and the Open/Closed String Duality, Int. J. Mod. Phys. A 20 (2005) 4699, arXiv:hep-th/0503156.

[14] M. Bochicchio, Glueball and Meson Spectrum in large-N QCD, In: Proceedings, Theory and Experiment for Hadrons on the Light-Front (Light Cone 2015), Few Body Syst. 57 (2016) 455, DOI: 10.1007/s00601-016-1100-6. 